\title{
Data Publish/Subscribe with Network Coding in DTN
}

\author{
http://dx.doi.org/10.3991/ijoe.v12i06.5512 \\ Xia $\mathrm{Yi}^{1}$, Deng Guanghong ${ }^{2}$ \\ ${ }^{1}$ Hubei Communications Technical College, Wuhan, China \\ ${ }^{2}$ Wuhan Digital Engineering Institute, Wuhan, China
}

\begin{abstract}
A data Publish/Subscribe method with network coding (DPS-NC) used in delay tolerant network is proposed. Based on the publish/subscribe mechanism, the topic data are encoded and transmitted using random network coding method, which can take full advantage of the network capacity to multicast. At the same time, for working out the problem of large data redundancy and vast invalid de-liveries in the network coding broadcast process, a packet routing scheme based on ant colony algorithm is brought forward. In the end, some simulation experiments are carried out and the results show that the DPS-NC method can get better delivery performance.
\end{abstract}

Index Terms-delay tolerant network, random network coding, ant colony algorithm, data dissemination.

\section{INTRODUCTION}

As a new transmission mode in Delay Tolerant Networks (DTN) [1, 2] network, network coding has been proved that it can improve network utilization, reduce transmission delay and enhance network security. R.Ahlswede, et al [3] proved that the bandwidth utilization can be increased by $33 \%$ for butterfly network. S Y Robert Li, et al [4] pointed out that for the general multicast network, using the linear network encoding can reach the multicast capacity. But in the DTN environment, the flood broadcast mechanism of network coding will bring out some problems such as information redundancy, invalid transmission, and data congestion [5].

The data publish/subscribe mechanism [6] was brought out for improving the data delivery efficiency and reducing information redundancy. It establish efficient and loose coupling of information distribution channels between data producers and consumers, to improve data transmission efficiency [7]. However, the data publish/subscribe mechanism was often used in reliable network environment [8]; the applications in DTN network, and particularly applications with network coding get few focus.

Direct at the demand of data distribution in DTN network, a network coding-based data publish/subscribe method (DPS-NC) is proposed. It introduce the network coding method into data transmission process to improve data delivery performance and reduce data redundancy. At the same time, it brought out a packet routing method based on ant colony algorithm to improve data delivery efficiency.

\section{DATA PUBLISH/SUBSCRIBE WITH NETWORK CODING}

\section{A. network coding based data transmission}

Assuming that the multicast rate of source is $h$, and the topic package $P=\left\{p_{1}, p_{2}, \ldots . p_{n}\right\}$ is distributed. When transmitting data packet $p_{1}$, it is discomposed to $\left\{p_{11}, p_{12}, \ldots, p_{1 h}\right\}$, encoded and transmitted to the output channel, the encoded value is $I(e)=e=(i, j), i=\operatorname{tail}(e), j=\operatorname{head}(e)$.

In node $k$, an encoding vector $n_{e}=\left(n_{e, 1}, n_{e, 2}, \ldots, n_{e, h}\right)$ is randomly generated for each of the output channel $k$. the $I(E)$ is the calculated value of the topic data with the encoding vector. $I(e)=\sum_{i=1}^{h} n_{e, i} x_{i}$, $\operatorname{tail}(e)=k$.

For every encoding vector in node's output channel $e$, $n_{e}=\left(n_{e, 1}, n_{e, 2}, \ldots, n_{e, h}\right)$, they compose a random network coding scheme. The destination received data $I(e)$ is the global encoded value calculated by several encoding nodes.

$I(e)=\sum_{i=1}^{h} g_{i} p_{i}, g_{i}=n_{e, i} M_{k, i}, n_{e, i}$ is the first $i$ element of encoding vector in output channel $e, M_{k, i}$ is the first $i$ input vector of node $k$. The encoding vector and encoded value in packets received by destination construct a matrix equation:

$$
\left[\begin{array}{llll}
g_{11} & g_{12} & \mathrm{~L} & g_{1 h} \\
g_{21} & g_{22} & \mathrm{~L} & g_{2 h} \\
& \mathrm{M} \mathrm{M} & \mathrm{O} & \mathrm{M} \\
g_{n 1} & g_{n 2} & \mathrm{~L} & g_{n h}
\end{array}\right]\left[\begin{array}{c}
x_{1} \\
x_{2} \\
\mathrm{M} \\
x_{h}
\end{array}\right]=\left[\begin{array}{c}
I_{1} \\
I_{2} \\
\mathrm{M} \\
I_{n}
\end{array}\right]
$$

According to the linear equations solvable conditions, if the coding vector matrix composed by $g_{i j}$ is full rank, then the data from source node can be decoded at sink.

\section{B. Data publication and subscription}

The source divides the distribute information into several topic packets, and then every topic packet is decomposed to a number of encoded packets according to the multicast rate, and published to neighbor nodes. In order to reduce the information redundancy in the network, the max survival time and the max non-subscribe time are set in every topic data, if it up to the max time, the topic packet will be picked out. The topic diffusion level is denoted by the max times of data packet relayed, the higher diffusion level, the more packet copies, and the more chance to establish topic publish/subscribe relationships, 


\section{DATA PUBlish/SUBSCRIBE WITH NETWORK CODING IN DTN}

but with the higher network load and information redundancy.

The destination generates topic subscription packets according to information demand, and diffuses them in the network to tell the nodes where are subscribing. For reducing data redundancy and delivery delay, the ant colony algorithm is introduced in guiding published topic packets delver to sinks with subscription pheromone. During the process of subscribe packet transmitting, the relay nodes will select the higher subscription pheromone road to delivery.

The Fig 1 and Fig 2 shows the publication packet format and the subscription packet format.

Take example of the Fig 3 , the source node $b$ publishes topic data $p$, the destination nodes $x, y$ subscribe it, $p$ is published to $2,3,5,4,9,6,8$, the subscription packets from $x, y$ transmit by nodes $7,11,12,1,8,9,6$, the publish/subscribe relationship is established at nodes $8,9,6$, and the topic data will transmit around the better subscription roads to sinks according to the subscription pheromone.

\section{C. routing of encoded packet based on ant colony algo- rithm}

For improving the data delivery efficiency and reducing data redundancy, the thought of ant colony algorithm is introduced in packets routing process, using the subscription pheromone to guide the published packet transmit around better subscription road to destinations.

The subscription pheromone comprehensively reflection the relay times, transmission delay and carry delay in data delivery process. The update strategy of the subscription pheromone is as follows:

$$
p_{j}\left(t p c_{-} d_{k}\right)=\left(p_{i}\left(t p c_{-} d_{k}\right)\left(1-\frac{t_{\text {delay }}}{\mathrm{TTL}_{t p c_{-} d_{k}}}\right)\right)^{\alpha}\left(1-\frac{\tau}{\mathrm{TTL}_{t p c_{-} d_{k}}}\right)
$$

$i, j, k \in V,(i, j) \in E$, the topic packet $t p c_{-} d_{k}$ published by node $k$ transmit from node $i$ to node $j, p_{i}\left(t p c_{-} d_{k}\right)$ and $p_{j}\left(t p c_{-} d_{k}\right)$ are the subscription pheromone of packet $t p c_{-} d_{k}$ at node $i$ and $j, t_{\text {delay }}$ is the carry delay of the packet at node $i, \mathrm{TTL}_{t p c_{-} d_{k}}$ is the max survival time of the packet, $\alpha=1 /$ topic diffusion level, $\tau$ is the transmit delay of packet transmit from node $i$ to $j$.

\section{SIMULATION EXPERIMENTS}

We designed a typical DTN network scenario in the simulation tool ONE[9], and simulated the Epidemic Routing method(ER), the Random Network Coding method(RNC), and the DPS-NC method introduced by this paper, and then analyzed the data delivery ratio, delay and data redundancy of the three methods.

The Fig 4, Fig 5 and Fig 6 are the analysis of the data delivery ratio, delay and data redundancy of the three methods with the change of node numbers.

In order to verify check the data delivery performance in the condition of network topology varies sharply. We analyzed the delivery ratio, delay and data redundancy with the changes of nodes moving speeds, the results are as shown in the Fig 7, Fig 8 and Fig 9.

As the simulation results show, compare with the ER and RNC method, the DPS-NC method obtained better delivery performance.

\begin{tabular}{|c|c|}
\hline Topic & Batch number \\
\hline The max survival time & Topic diffuse level \\
\hline The max no-subscription time \\
\hline \multicolumn{2}{|c|}{ Encoding vector } \\
\hline \multicolumn{2}{|c|}{ Encoded data } \\
\hline
\end{tabular}

Figure 1. Format of topic publication packet

\begin{tabular}{|c|c|}
\hline Topic & The max survival time \\
\hline The subscription pheromone \\
\hline Relay nodes \\
\hline$\ldots \ldots$ \\
\hline$\ldots \ldots$ \\
\hline
\end{tabular}

Figure 2. Format of topic subscription packet

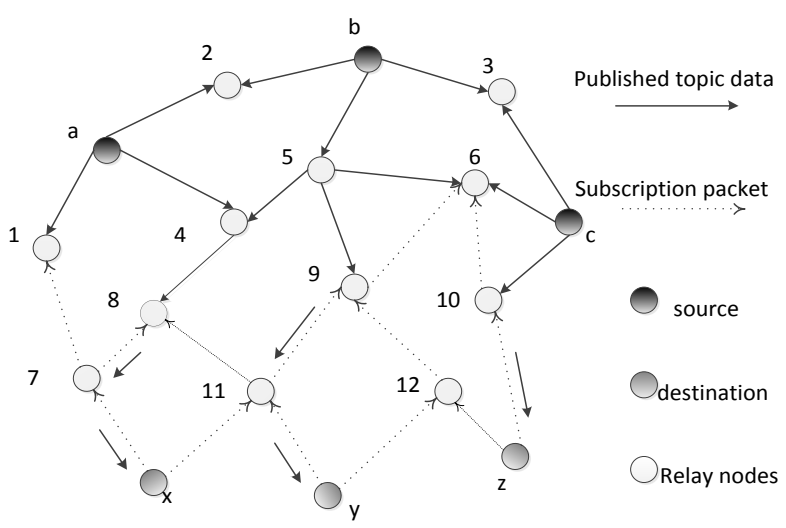

Figure 3. Sketch map of data publish/subscribe with DPS-NC method

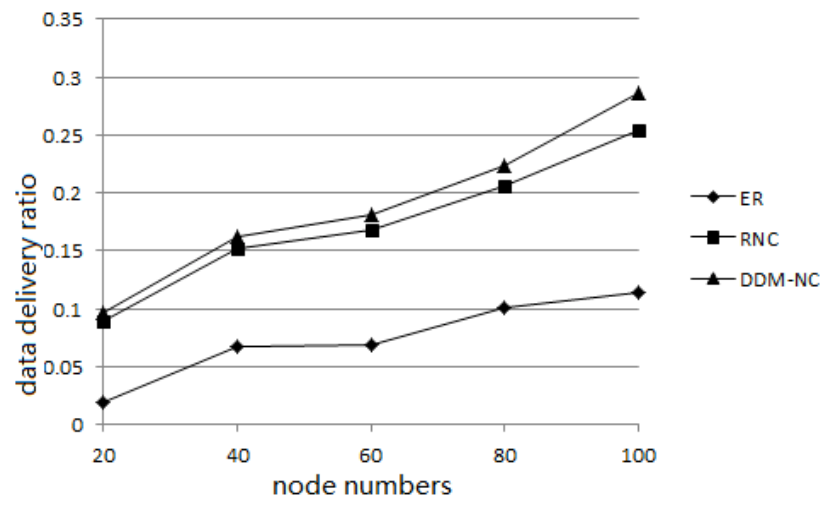

Figure 4. Comparison of data delivery ratio with node numbers

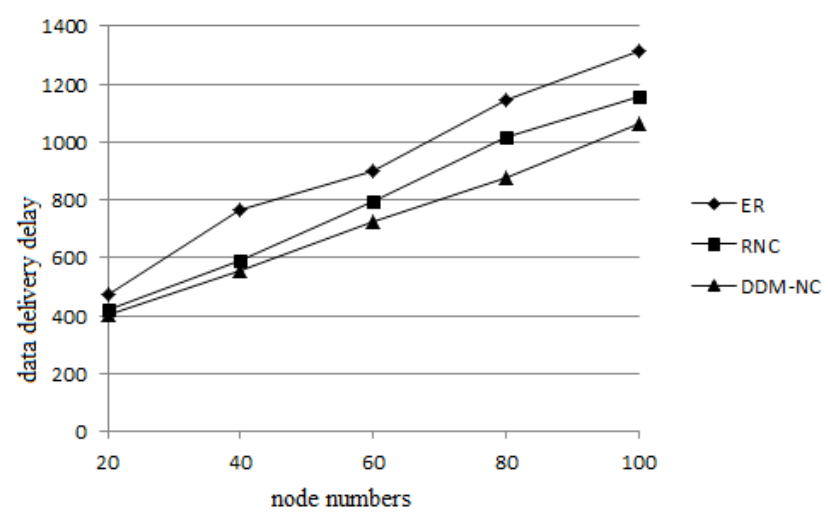

Figure 5. Comparison of data delivery delay with node numbers 


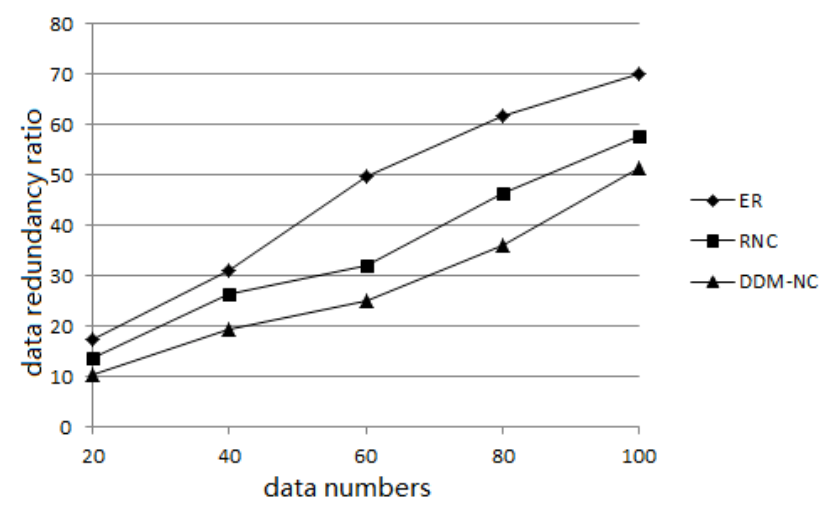

Figure 6. Comparison of data redundancy ratio with node numbers

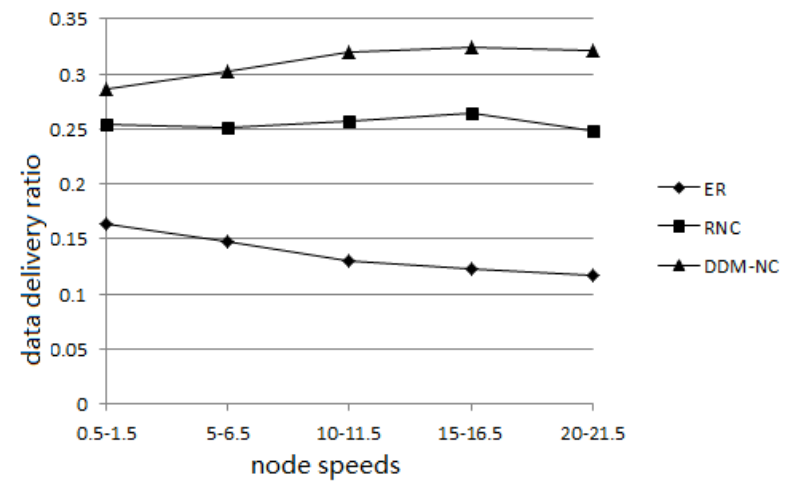

Figure 7. Comparison of data delivery ratio with speed changes

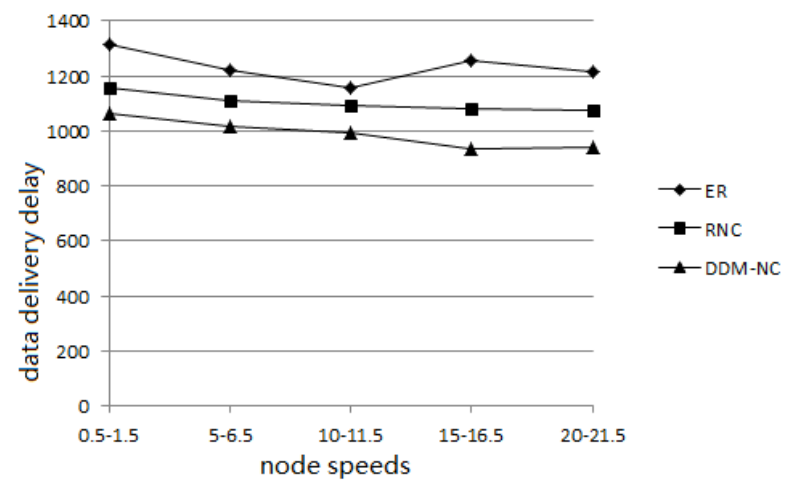

Figure 8. Comparison of data delivery delay with speed changes

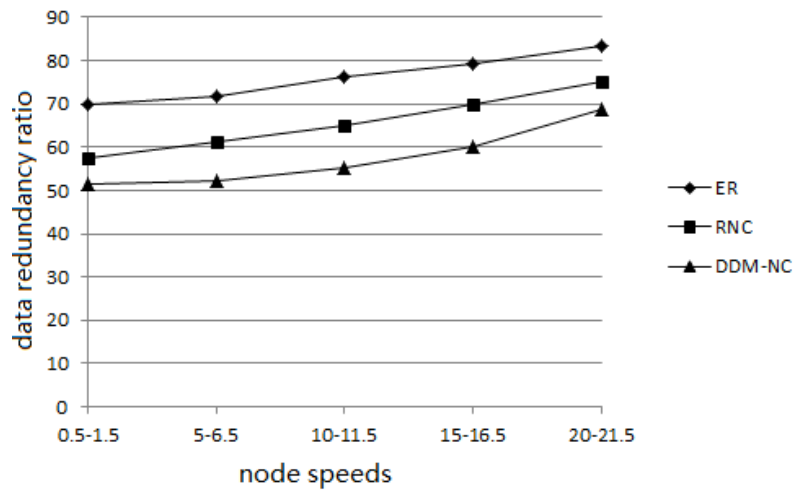

Figure 9. Comparison of data redundancy ratio with speed changes

\section{Conclusions S}

Combined with the network encoding and data publish/subscribe mechanism, it proposed a network coding based data distribution method with ant colony algorithm for DTN environment. For improving the data distribution performance, the network coding method is intruded into the data publish/subscribe mechanism, and using the ant colony algorithm for packet routing to improve the delivery performance. The method makes up for the shortcomings of network utilization in-sufficient and large risk of data re-transmission in traditional DTN routing method, and remedies the defect of large data redundancy, invalid delivery, and low delivery efficiency of random network encoding method. The simulation results show that the method can get better data delivery performance.

\section{REFERENCES}

[1] Fall K, "A delay tolerant network architecture for challenged internets." In Proc of the 2003 Conf on Applications, Technologies, Architectures and Protocols for Computer Communications, Karlsruhe, Germany: ACM, 2003, pp.27-34. http://dx.doi.org/10.1145/863955.863960

[2] Y P Xiong, L M Sun, J W Niu, et al. "Opportunistic networks". In Journal of Software. vol 20, 2009, pp.124-137. http://dx.doi.org/10.3724/SP.J.1001.2009.00124

[3] Ahlswede R., C Ning, S Li, et al. "Network information flow" in IEEE Transactions on Information Theory. Vol 46, 2000, pp.1204-1216. http://dx.doi.org/10.1109/18.850663

[4] S Y Robert Li, Raymond W Yeung, N Cai. "Linear network coding" in IEEE Transactions on Information Theory, vol 49,2003, pp.371-381. http://dx.doi.org/10.1109/TIT.2002.807285

[5] B C Li, D Niu. "Random network coding in Peer-to-Peer networks: from theory to practice" in Proceedings of the IEEE. Vol 99, 2011, pp.513-523. http://dx.doi.org/10.1109/JPROC.2010.2091930

[6] Eugster P T, Felber P A, Guerraoui K Kermarrec A M. "The many faces of publish/subscribe" in ACM Computing Surveys. Vol 35, 2003, pp.114-131. http://dx.doi.org/10.1145/857076.857078

[7] J Q Zhu, M Liu, H G Gong, et al. "Event delivery in publish/subscribe system for delay tolerant sensor networks" in Journal of Software. vol 21, 2010, pp.1954-1967. http://dx.doi.org/10.3724/SP.J.1001.2010.03583

[8] J S Su, Q L Hu, B K Zhao, et al. "Routing techniques on delay/disruption tolerant networks" in Journal of Software. vol 21, 2010 ,

pp.119-132. http://dx.doi.org/10.3724/SP.J.1001.2010.03689

[9] Ari Keränen, Jörg Ott, Teemu Kärkkäinen. "The ONE Simulator for DTN Protocol Evaluation" in SIMUTools 2009. Rome, Italy: ICST, 2009.

\section{AUTHORS}

XIA Yi received the Master's degree in Computer Science and Technology from Wuhan University of Science and Technology, in 2007. She is currently a senior teacher at Institute of Traffic Information, Hubei Communications Technical College. Her research interests are broadly on delay tolerant network and random network coding (Email: stephenden@163.com)

DENG Guanghong received the Ph.D. degree in Computer Science and Technology from Harbin Engineering University, Harbin, in 2015.

$\mathrm{He}$ is currently a Senior Engineer at the Department of Scientific Research on System, Wuhan Digital Engineering Institute. His research interests are broadly on experimental wireless networking and delay tolerant network.

Submitted 23 January 2016. Published as resubmitted by the authors 25 March 2016. 